\title{
CuAu Structure in the Restricted Primitive Model and Oppositely Charged Colloids
}

\author{
A.-P. Hynninen, ${ }^{*}$ M.E. Leunissen, ${ }^{*}$ A. van Blaaderen, and M. Dijkstra \\ Soft Condensed Matter, Debye Institute, Utrecht University, Princetonplein 5, 3584 CC Utrecht, The Netherlands
}

(Received 23 August 2005; published 4 January 2006)

\begin{abstract}
We study the phase behavior of oppositely charged equal-size hard spheres both theoretically and experimentally, using Monte Carlo simulations and confocal microscopy. In the simulations, two systems are considered: the restricted primitive model (RPM) and a system of screened Coulomb particles. We construct the phase diagrams of both systems by computer simulations and predict a novel solid phase that has the $\mathrm{CuAu}$ structure. In addition, the $\mathrm{CuAu}$ structure is observed experimentally in a system of oppositely charged colloids. The qualitative agreement between the RPM, the screened Coulomb system, and the experiments shows that colloids form a suitable model system to study phase behavior in ionic systems.
\end{abstract}

A basic understanding of phenomena like gas-liquid condensation or freezing of atoms and molecules can be often acquired by studying model systems, in which the interactions between the particles are simplified to the bare minimum. For instance, hard spheres are often used to represent systems with repulsive interactions [1], whereas charged hard spheres are commonly employed to model ionic systems. The simplest and best-known model for the latter is the restricted primitive model (RPM), which consists of a binary mixture of equal-size hard spheres suspended in a uniform continuum solvent, half of which carry a negative and the other half a positive charge of equal magnitude. In the past, the phase behavior of the RPM has been studied extensively [2-5], and the global phase diagram, which includes fluid, $\mathrm{CsCl}$, face-centered-cubic (fcc) disordered, and "tetragonal" phases, has been constructed. Interestingly, all these phases, except the tetragonal phase, have recently also been observed experimentally in a system of oppositely charged colloids [6,7]. The phase behavior of this system can be understood on the basis of screened Coulomb potentials, in which the screening is due to the presence of co- and counterions in the solvent. In this Letter, we present experiments on oppositely charged colloids that display a novel crystal structure where the particles are arranged in a CuAu-type crystal. In order to check the stability of this structure, we calculate the phase diagram of particles interacting via screened Coulomb potentials, and reexamine the phase behavior of the RPM. We find that the two phase diagrams are qualitatively similar, and, more importantly, that both contain the novel $\mathrm{CuAu}$ structure.

Our simulations consist of $N$ spheres with a diameter $\sigma$ in a volume $V$, half of which carry a positive charge $Z e$ and the other half a negative charge $-Z e$. We assume the Derjaguin-Landau-Verwey-Overbeek (DLVO) screened Coulomb pair potential, which is written as [8]

$$
\frac{u(r)}{k_{B} T}=\left\{\begin{array}{ll} 
\pm \frac{Z^{2}}{(1+\kappa \sigma / 2)^{2}} \frac{\lambda_{B}}{\sigma} \frac{e^{-\kappa(r-\sigma)}}{r / \sigma} & r \geq \sigma \\
\infty & r<\sigma
\end{array},\right.
$$

where the sign depends on the particle charges, $r$ is the distance between the particles, $\lambda_{B}=e^{2} / \epsilon_{s} k_{B} T$ is the Bjerrum length, $\epsilon_{s}$ is the dielectric constant of the solvent, $\kappa=\sqrt{8 \pi \lambda_{B} \rho_{\text {salt }}}$ is the inverse Debye screening length, and $\rho_{\text {salt }}$ is the salt concentration. van der Waals forces are negligible in our system [6]. We define a packing fraction $\eta=\pi \sigma^{3} N / 6 V$ and a reduced temperature $T^{*}=$ $(1+\kappa \sigma / 2)^{2} \sigma / Z^{2} \lambda_{B}$ that is the inverse of the contact value of the potential in Eq. (1). The RPM is achieved by setting $\kappa=0$, because then the screened Coulomb potential in Eq. (1) reduces to the Coulomb potential and $T^{*}$ to the dimensionless temperature of Refs. [2-5].

We performed Monte Carlo (MC) simulations with the potential in Eq. (1) using the canonical $(N V T)$ ensemble and periodic boundary conditions [9]. The interactions were truncated at one half of the smallest box side length, $L_{\min }$. In the case of the RPM, we used the Ewald summation method $[9,10]$ with $k$-space cutoff at $k_{\text {cut }}=10 \pi / L_{\min }$. Our Ewald summation method was tested to reproduce the Madelung energies in Ref. [4] for the $\mathrm{CsCl}, \mathrm{CuAu}$, and tetragonal structures. We did our simulations for the screened Coulomb particles at an inverse screening length $\kappa \sigma=6$, which approximately corresponds to our experimental system (see below). We looked for possible crystal structure candidates by a simulated annealing method similar to that used in Ref. [4], but included also MC moves that change the shape of the simulation box and performed the runs at various packing fractions below close packing. The same crystal phases were found as the ones reported in Ref. [4]: $\mathrm{CsCl}$, fcc disordered, tetragonal, $\mathrm{CuAu}$, and the hexagonal-close-packed (hcp) counterparts of the tetragonal and $\mathrm{CuAu}$ structures. We found that the hcp-CuAu phase is metastable with respect to $\mathrm{CuAu}$, and that the free energy of the hcp-tetragonal phase is, within our error estimates, equal to the tetragonal phase, but definitely becomes metastable at close packing. The overall symmetry (of both the positive and the negative particles) of a CsCl-type crystal is body-centered cubic (bcc) and the substitutional order is shown in Fig. 1(a). In the 


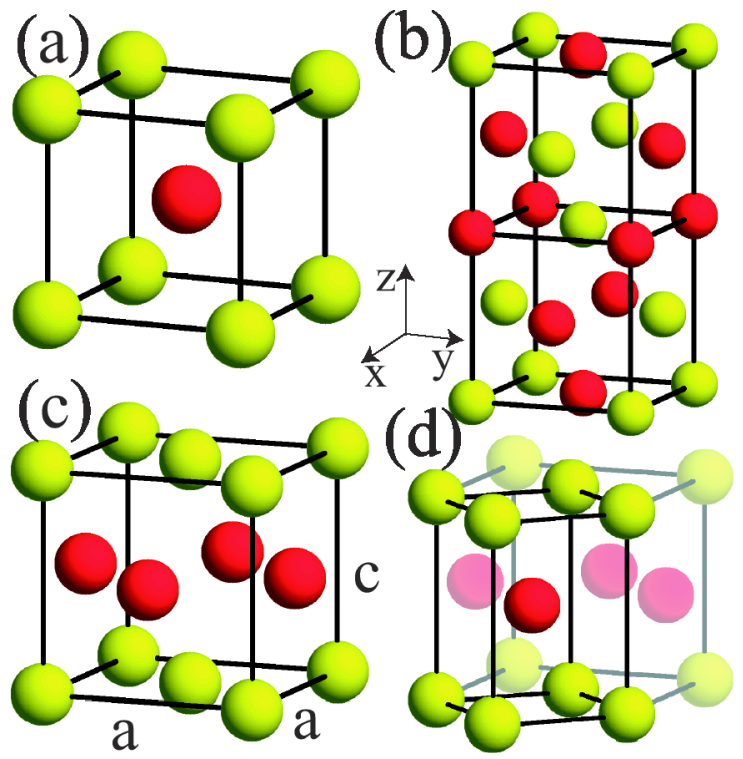

FIG. 1 (color online). Unit cells of (a) $\mathrm{CsCl}$, (b) tetragonal, and (c)-(d) $\mathrm{CuAu}$ structure, where dark (red) and light (yellow) spheres have opposite charges. In (d), the tetragonal cell of the $\mathrm{CuAu}$ is highlighted.

tetragonal phase, the overall symmetry is face-centered tetragonal (fct) and the substitutional order can be described by two fct cells on top of each other, as shown in Fig. 1(b). The fcc disordered phase has no substitutional order and the two particle species sit on an fcc lattice. Finally, in the $\mathrm{CuAu}$ structure, the overall crystal symmetry is fct and the oppositely charged particles are arranged in alternating layers as shown in Fig. 1(c). We found that the $\mathrm{CsCl}-\mathrm{CuAu}$ transition is a weakly first-order martensitic phase transition [11] and, therefore, we defined an order parameter $a / c$, which is the ratio of the $\mathrm{CuAu}$ unit cell side lengths; see Fig. 1(c). In order to understand this martensitic transition, it is useful to consider the tetragonal cell of the $\mathrm{CuAu}$ structure shown in Fig. 1(d). In Fig. 1(d), the dark (red) particle corresponds to the center particle of the $\mathrm{CsCl}$ unit cell in Fig. 1(a). The $\mathrm{CuAu}$ to $\mathrm{CsCl}$ transformation occurs by a continuous deformation of the tetragonal cell from $a / c=1$ to $a / c=\sqrt{2}$, where the cell has become cubic. To distinguish between the two structures, we define the threshold value to be in between, i.e., $a / c=$ $(1+\sqrt{2}) / 2 \approx 1.2$. The phase diagrams were determined from the Helmholtz free energies that were calculated for the fluid, $\mathrm{CsCl}, \mathrm{CuAu}$, and tetragonal phases using thermodynamic integration methods $[9,12]$. In the thermodynamic integration of the fluid phase, we used the hardsphere fluid as a reference state, whereas in the solid phases, the reference state was the Einstein crystal. The numerical integrations were done using a 10-point Gaussian quadrature and the ensemble averages were calculated from runs with $40000 \mathrm{MC}$ cycles (attempts to displace each particle once), after first equilibrating the system during $20000 \mathrm{MC}$ cycles. For the $\mathrm{CuAu}$ and te- tragonal crystals, a tetragonally shaped simulation box was used. We determined the shape of the simulation box in a separate MC run with additional shape moves that change the side lengths of the box while conserving the volume.

Experimentally, we established a system of oppositely charged particles by preparing salt-containing mixtures of differently labeled fluorescent polymethylmethacrylate (PMMA) colloids in a mixture of cyclohexyl bromide (Fluka) and cis-decalin (27.2 w/w, Sigma-Aldrich), as described in [6]. This solvent mixture nearly matches the density and refractive index of the particles and has a dielectric constant of $\epsilon_{s}=5.59\left(\lambda_{B} \approx 10 \mathrm{~nm}\right)$. The particles were made by dispersion polymerization, covalently labeled with the fluorophore 7-nitrobenzo-2-oxa-1,3diazol (NBD) or rhodamine isothiocyanate (RITC) and sterically stabilized with poly-12-hydroxystearic acid [13]. The NBD- and RITC-labeled particles had diameters of $\sigma=1.98$ and $2.16 \mu \mathrm{m}$, respectively, i.e., the size ratio was close to unity (0.92). The size polydispersity was in both cases 3\%, as determined with light scattering and electron microscopy. The suspensions were 1:1 number density mixtures at an overall packing fraction $\eta \approx 0.30$. The particle charge and the range of the electrostatic interactions were tuned by adding $\sim 190 \mu \mathrm{M}$ of the salt tetrabutylammonium bromide (Sigma-Aldrich). Under these conditions the NBD-labeled particles were negatively charged, whereas the RITC-labeled particles were positive. We estimated $\kappa \sigma \approx 8 \pm 2$ from conductivity measurements on the particle-free solvent mixture (with a Scientifica 627 conductivity meter) and applying Walden's rule [14]. We used $2.217 \mathrm{cP}$ for the viscosity of the solvent mixture (measured at $25^{\circ} \mathrm{C}$ with a Schott ViscoSystem). In our previous work, we determined the charges of the same particles, but under conditions at which they formed CsCl-type crystals [6]. We then found charges of +110 e and -75 e; i.e., the charge ratio is close to unity $(\sim 1.5)$. Although we could not determine the exact electrophoretic mobilities of the particles in the dense suspensions investigated here, semiquantitative observations indicate that the charges were likely lower. Therefore, we estimate the reduced temperature to be $T^{*} \geq$ 0.6 in the present experimental system.

Figures 2 and 3 show the theoretical phase diagrams of the RPM and the screened Coulomb particles, respectively, in the packing fraction $\eta$-reduced temperature $T^{*}$ representation. The phase coexistence regions were determined from the Helmholtz free energies using a common tangent construction. In Fig. 2, we only calculated the $\mathrm{CsCl}-\mathrm{CuAu}$ and $\mathrm{CuAu}$-tetragonal phase lines, as the other parts of the phase diagram are known from earlier work $[5,15,16]$. In Fig. 3, the weakly first-order tetragonal/CuAu-fcc disordered phase line [17] was obtained from the jump in the internal energy $U$ that occurs when crossing the phase boundary [4]. The same method was also used for the $\mathrm{CsCl}-\mathrm{fcc}$ disordered phase transition, although the order 


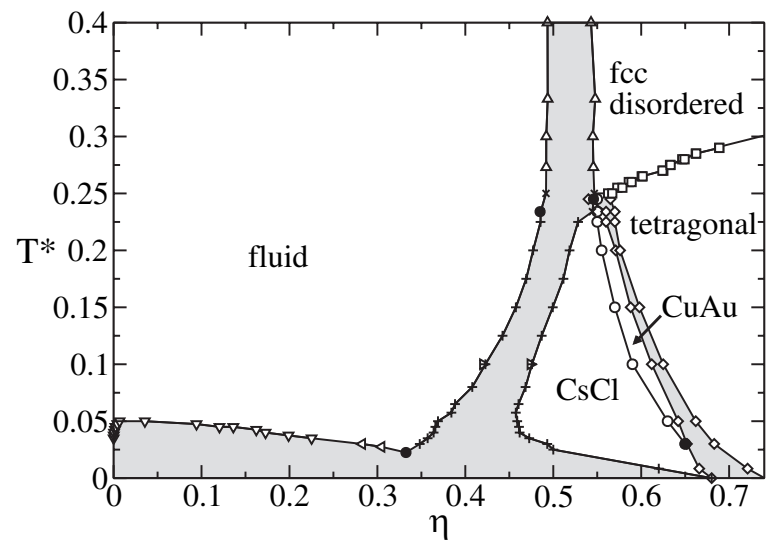

FIG. 2. Phase diagram of the RPM in the packing fraction $\eta$-reduced temperature $T^{*}$ plane. Phase lines for fluid-CsCl $(+)$, fluid-fcc disordered $(\triangle)$, fluid-tetragonal $(\times)$, and fcc disordered-tetragonal $(\square)$ are from Ref. [5]. Gas-liquid phase envelope $(\nabla)$ is from Ref. [16] and liquid points $(\triangleleft)$ are from Ref. [15]. The circles $(\bigcirc)$ and diamonds $(\diamond)$ mark the CsCl$\mathrm{CuAu}$ and $\mathrm{CuAu}$-tetragonal phase transitions, respectively. The black circles (1) mark the position of the triple points and the gray areas denote the coexistence regions where tie lines are horizontal. The lines are guides to the eye.

of this phase transition has not yet been well characterized. Qualitatively, the two phase diagrams are quite similar. Both have a fluid phase in the low packing fraction region, a fluid-fcc disordered phase coexistence in the high temperature-high packing fraction region, a broad gas$\mathrm{CsCl}$ phase coexistence in the low temperature region, and, in the intermediate temperature region, a sequence of $\mathrm{CsCl}, \mathrm{CuAu}$, and tetragonal phases with increasing packing fraction. A comparison between the two phase diagrams shows that screening enlarges the $\mathrm{CuAu}$ region. In the RPM phase diagram, the $\mathrm{CuAu}$ phase forms a narrow

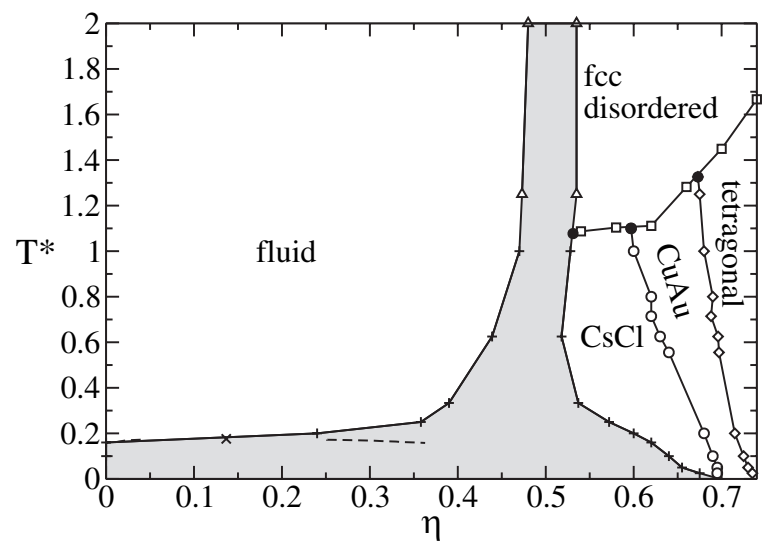

FIG. 3. Phase diagram of the screened Coulomb particles with inverse Debye screening length $\kappa \sigma=6$ in the packing fraction $\eta$-reduced temperature $T^{*}$ plane. The dashed line and the cross $(\times)$ show the metastable gas-liquid phase envelope and the critical point, respectively. The rest of the symbols are as in Fig. 2 (but not given in the references cited there). pocket that has a low- $T^{*}$ triple point and seems to close up before reaching the fcc disordered region. In the screened Coulomb phase diagram, however, the $\mathrm{CuAu}$ pocket is broad and extends from $T^{*}=0$ all the way to the fcc disordered region at $T^{*} \approx 1.2$. This makes it possible to observe $\mathrm{CuAu}-\mathrm{fcc}$ disordered phase coexistence, which may explain the experimental observations (see below). We like to point out that the RPM and the screened Coulomb phase diagrams display a slightly different behavior in the $T^{*} \rightarrow 0$ limit: although both exhibit a broad gas-CsCl coexistence at $\eta<0.68$, at higher $\eta$ the RPM has a stable tetragonal phase, whereas the screened Coulomb system has a stable $\mathrm{CuAu}$ phase, only to be followed by the tetragonal phase at $\eta \gtrsim 0.73$. Screening also affects the stability of the gas-liquid critical point. The RPM has a stable gas-liquid critical point at $T^{*} \approx 0.05$, but in the screened Coulomb phase diagram, the critical point is metastable with respect to the gas- $\mathrm{CsCl}$ phase coexistence; see Fig. 3. According to our preliminary results, the gasliquid critical point of the screened Coulomb system is stable at $\kappa \sigma \lesssim 4$; see also [18].

We studied our system of oppositely charged colloids by means of confocal laser scanning microscopy. Figure 4 shows a selection of representative confocal images, where the packing fraction is $\eta=0.58 \pm 0.04$. The crystalline structures were observed within 18 days after sample

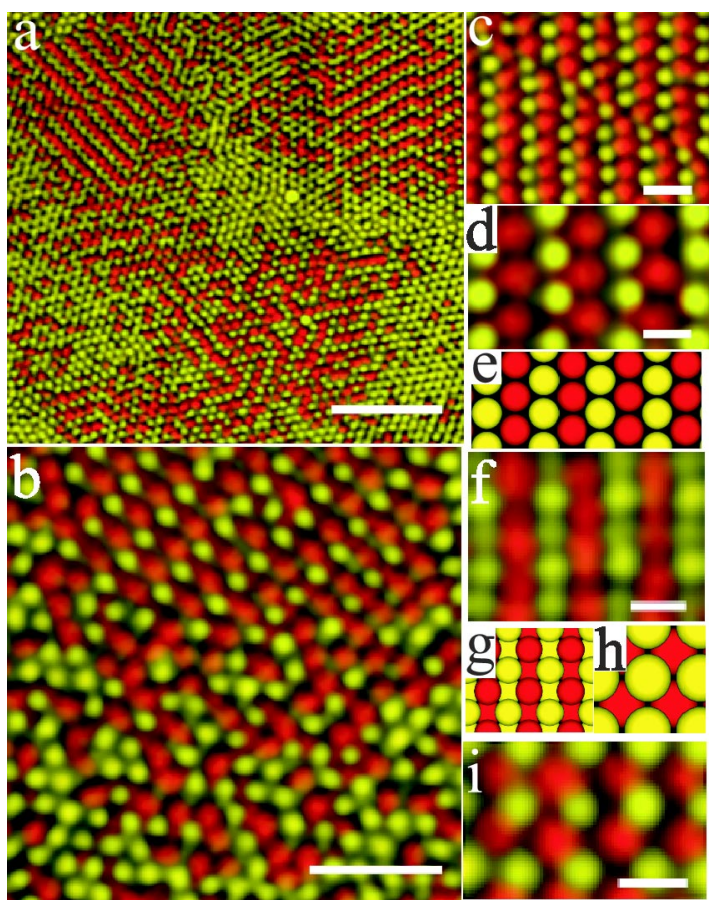

FIG. 4 (color online). Confocal microscope images of $\mathrm{CuAu}-$ type crystallites: (a), (b) in the presence of an rhcp disordered crystal, (c), (d) a (111) plane, (f) a (100) plane, (i) a (001) plane. In (c), one can observe a line defect. Illustrations of CuAu-type crystal planes: (e) the (111) plane, (g) the (100) plane, and (h) the (001) plane. The scale bars are: $25 \mu \mathrm{m}$ in (a), $10 \mu \mathrm{m}$ in (b), $5 \mu \mathrm{m}$ in (c), and $2.5 \mu \mathrm{m}$ in (d), (f), and (i). 
preparation and they remained stable for months. In order to check that they indeed consist of oppositely charged particles, we subjected them to a static external electric field $(7.5 \mathrm{~V} / \mathrm{mm})$. This caused the two particle species to move in opposite directions, thereby melting the structures. Figures 4(a) and 4(b) show the CuAu phase in the presence of a random-hexagonal-close-packed (rhcp) disordered phase. The $\mathrm{CuAu}$ structure is easily recognized as it has alternating stripes of dark (red) and bright (yellow) particles that make up the (111) hexagonal plane [with respect to the orientation of the unit cell shown in Fig. 1(c)]. Remarkably, whereas the CuAu crystallites were strictly fcc, the substitutionally disordered structures were rhcp. Note that on the basis of hard spheres, one expects to see rhcp because of the small free energy difference between fcc and hcp crystals [19]. The simultaneous observation of the $\mathrm{CuAu}$ and fcc disordered phases agrees with the screened Coulomb phase diagram in Fig. 3, in which we find the $\mathrm{CuAu}$ and fcc disordered phases connected by a weakly first-order phase line. Unfortunately, a more quantitative comparison between the simulations and the experiments is difficult as it is not known whether the experimentally observed structures are in equilibrium, and the experimental values of $\kappa \sigma$ and $T^{*}$ are not known accurately. However, the relatively low packing fraction $(\eta \approx 0.58)$ of the observed $\mathrm{CuAu}$ phase and the presence of the rhcp disordered phase point towards the intermediate temperature region of the phase diagram in Fig. 3 where $T^{*} \approx 1$. Thus, the experiments fall in between the energy $\left(T^{*} \ll 1\right)$ and entropy $\left(T^{*} \gg 1\right)$ dominated limits. Figures 4(c) and 4(d) show close up images of the (111) plane, now with the stripes running vertically. Figure 4(e) illustrates the (111) plane in an ideal crystal. Although most of the CuAu-type crystallites were oriented with their (111) plane parallel to the bottom wall of the sample cell, we were able to observe (100) and (001) planes as well; see Figs. 4(f)-4(i). As can be seen from Fig. 4(f) [and Fig. 4(g)], the (100) plane consists of alternating layers of oppositely charged particles. Finally, we like to point out that the crystalline structures described above consisted of approximately 10 layers of particles and always formed near the bottom of the sample cell. This means that these structures need a higher osmotic pressure to form.

To conclude, we constructed the phase diagram of screened Coulomb particles and reexamined the phase behavior of the RPM. We showed that the two phase diagrams are qualitatively similar, and more importantly, that both contain a novel solid phase which is analogous to the $\mathrm{CuAu}$ structure. We also observed the $\mathrm{CuAu}$ structure in our experiments with oppositely charged colloids, which can be seen as an experimental realization of screened Coulomb particles. The fact that the experimental system is in the intermediate temperature region $\left(T^{*} \approx 1\right)$, that is, between the energy and entropy dominated limits, makes the observations of the $\mathrm{CuAu}$ structure even more interesting. It shows that a novel solid phase, distinct from the previously proposed $\mathrm{CsCl}, \mathrm{NaCl}$, and fcc disordered phases [6,7], can be found in this region. This has important implications for the search for new binary structures, which for oppositely charged colloids has so far only been carried out at zero temperature and zero pressure [20]. Furthermore, our results demonstrate that oppositely charged colloids can give insight into the phase behavior of the RPM, and show that colloids can be used for detailed studies, on the single particle level, of ionic phase transitions.

The authors acknowledge A. Fortini for the gas-liquid phase envelope in Fig. 3, R. P. A. Dullens and D. Derks for particle synthesis, A.D. Hollingsworth for solvent characterization, and C. Valeriani and D. Frenkel for useful discussions. This work is part of the research program of the "Stichting voor Fundamenteel Onderzoek der Materie (FOM)," which is financially supported by the "Nederlandse Organisatie voor Wetenschappelijk Onderzoek (NWO)."

*These authors contributed equally to this work.

[1] W. W. Wood and J. D. Jacobson, J. Chem. Phys. 27, 1207 (1957); B. J. Alder and T.E. Wainwright, ibid. 27, 1208 (1957).

[2] A.Z. Panagiotopoulos, J. Phys. Condens. Matter 17, S3205 (2005).

[3] B. Smit et al., Mol. Phys. 87, 159 (1996).

[4] F. Bresme et al., Phys. Rev. Lett. 85, 3217 (2000).

[5] C. Vega et al., J. Chem. Phys. 119, 964 (2003).

[6] M. E. Leunissen et al., Nature (London) 437, 235 (2005).

[7] P. Bartlett and A. I. Campbell, Phys. Rev. Lett. 95, 128302 (2005).

[8] B. V. Derjaguin and L. Landau, Acta Physicochim. URSS 14, 633 (1941); E. J. W. Verwey and J. Th. G. Overbeek, Theory of the Stability of Lyotropic Colloids (Elsevier, New York, 1948).

[9] D. Frenkel and B. Smit, Understanding Molecular Simulations (Academic, New York, 2002), 2nd ed.

[10] P. P. Ewald, Ann. Phys. (Leipzig) 64, 253 (1921).

[11] A. G. Khachaturyan, Theory of Structural Transformations in Solids (Wiley, New York, 1983).

[12] D. Frenkel and A. J. C. Ladd, J. Chem. Phys. 81, 3188 (1984).

[13] G. Bosma et al., J. Colloid Interface Sci. 245, 292 (2002).

[14] C. P. Royall et al., J. Phys. Condens. Matter 15, S3581 (2003).

[15] C. Vega et al., Phys. Rev. E 54, 2746 (1996).

[16] G. Orkoulas and A.Z. Panagiotopoulos, J. Chem. Phys. 101, 1452 (1994).

[17] J. L.F. Abascal et al., Phys. Rev. E 68, 052501 (2003).

[18] J. B. Caballero et al., J. Chem. Phys. 121, 2428 (2004).

[19] A. D. Bruce et al., Phys. Rev. Lett. 79, 3002 (1997).

[20] A.-P. Hynninen et al. (to be published). 